University of Nebraska - Lincoln

DigitalCommons@University of Nebraska - Lincoln

Role of Covalent Fe-As Bonding in the Magnetic Moment Formation and Exchange Mechanisms in Iron-Pnictide Superconductors

\author{
Kirill D. Belashchenko \\ University of Nebraska-Lincoln, belashchenko@unl.edu \\ Vladimir P. Antropov \\ Ames Laboratory, US Department of Energy, antropov@ameslab.gov
}

Follow this and additional works at: https://digitalcommons.unl.edu/cmrafacpub

Part of the Nanoscience and Nanotechnology Commons

\footnotetext{
Belashchenko, Kirill D. and Antropov, Vladimir P., "Role of Covalent Fe-As Bonding in the Magnetic Moment Formation and Exchange Mechanisms in Iron-Pnictide Superconductors" (2008). Faculty Publications from Nebraska Center for Materials and Nanoscience. 90.

https://digitalcommons.unl.edu/cmrafacpub/90
}

This Article is brought to you for free and open access by the Materials and Nanoscience, Nebraska Center for (NCMN) at DigitalCommons@University of Nebraska - Lincoln. It has been accepted for inclusion in Faculty Publications from Nebraska Center for Materials and Nanoscience by an authorized administrator of DigitalCommons@University of Nebraska - Lincoln. 


\title{
Role of covalent Fe-As bonding in the magnetic moment formation and exchange mechanisms in iron-pnictide superconductors
}

\author{
K. D. Belashchenko \\ Department of Physics and Astronomy and Nebraska Center for Materials and Nanoscience, University of Nebraska-Lincoln, Lincoln, \\ Nebraska 68588, USA \\ V. P. Antropov \\ Condensed Matter Physics, Ames Laboratory, Ames, Iowa 50011, USA
}

(Received 23 October 2008; published 19 December 2008)

\begin{abstract}
The electronic origin of the huge magnetostructural effect in layered Fe-As compounds is elucidated using $\mathrm{LiFeAs}$ as a prototype. The crucial feature of these materials is the strong covalent bonding between $\mathrm{Fe}$ and As, which tends to suppress the exchange splitting. The bonding-antibonding splitting is very sensitive to the distance between $\mathrm{Fe}$ and As nuclei. We argue that the fragile interplay between bonding and magnetism is universal for this family of compounds. The exchange interaction is analyzed in real space, along with its correlation with covalency and doping. The range of interaction and itinerancy increase as the Fe-As distance is decreased. Superexchange makes a large antiferromagnetic contribution to the nearest-neighbor coupling, which develops large anisotropy when the local moment is not too small. This anisotropy is very sensitive to doping.
\end{abstract}

DOI: 10.1103/PhysRevB.78.212505

PACS number(s): 75.30.Et, 74.25.Ha

Layered iron-pnictide compounds have recently attracted a lot of interest due to their high superconducting transition temperature. ${ }^{1}$ Pairing is widely believed to be mediated by spin fluctuations in these materials; ${ }^{2}$ understanding of magnetic interaction is therefore of utmost importance. Numerous first-principles calculations revealed a huge magnetostructural effect manifesting itself, in particular, in the strong sensitivity of the Fe local moments to the Fe-As distance $R_{\mathrm{Fe}-\mathrm{As}}{ }^{3}$

Tight-binding parametrizations of the band structure indicate that Fe-As hybridization is significant, ${ }^{4,5}$ and it was used to explain the instability of the $\mathrm{Fe}$ local moments ${ }^{5,6}$ (a similar effect is known for zinc-blende iron pnictides $\left.{ }^{7}\right)$. However, $a b$ initio results have been mainly described in terms of weak ${ }^{8,9}$ $\mathrm{Fe}$-As hybridization comparable to oxides. ${ }^{10}$ Strong Fe-As mixing was mentioned, ${ }^{3,11}$ but its role in the magnetism was not explained. Yildirim ${ }^{9}$ focused on the effect of the local moment on As-As bonding. In this Brief Report we show that the coupling between the local moment and the Fe-As distance is controlled by strong covalent Fe-As bonding, and we analyze its effects on the exchange interaction in the (likely ground-state) "stripe" phase using the linear-response technique. The salient features of chemical bonding and its relation to magnetism appear to be universal across the whole family of iron-pnictide layered materials, and we chose $\mathrm{LiFeAs}$, which is a superconductor below $18 \mathrm{~K},{ }^{12}$ as a representative example.

The band structure of LiFeAs was calculated by Singh, ${ }^{13}$ in most respects it is similar to $\mathrm{LaFeAsO}, \mathrm{BaFe}_{2} \mathrm{As}_{2}$, and other members of this layered iron-pnictide family. Let us analyze the orbital content of the Bloch states. Figure 1(a) shows the energy bands ${ }^{14}$ of nonmagnetic LiFeAs calculated for the experimental structure. ${ }^{15}$ In this picture, As $(4 p)$ weight is shown in red color and also using line thickness; the iron $(4 s$ and $3 d)$ weight is shown in blue. We have verified that the weights of different $3 d$ cubic harmonics on $\mathrm{Fe}$ sites (not shown) are similar to those plotted in Ref. 8 for $\mathrm{LaFeAsO}$. It is seen from Fig. 1(a) that As and Fe states form fully mixed bonding and antibonding states centered, respectively, at $3.5 \mathrm{eV}$ below and at $1.5 \mathrm{eV}$ above the Fermi level $E_{F}$. The large bonding-antibonding splitting of about $5 \mathrm{eV}$ indicates very strong covalent bonding between As $p$ and Fe $d$ states in the sense that the splitting is large compared to the bare level separation. The hybridized bands have almost equal weights of $\mathrm{Fe}$ and As states, and the As states contribute equally to the bonding and antibonding states. This picture is somewhat different from that presented for $\mathrm{LaFeAsO},{ }^{11}$ where the bands were divided in an upper group of mainly Fe $3 d$ bands and a lower group of mainly pnictogen and oxygen $p$ bands. The separation of As states in two subbands can only be explained by hybridization with $\mathrm{Fe}$. Indeed, the As states of a fictitious system with $\mathrm{Fe}$ atoms removed from the lattice form a gapless set of bands about 4 $\mathrm{eV}$ wide; this was checked using the full-potential linear augmented plane-wave (FLAPW) method. ${ }^{14}$ We also note that although the bare $\mathrm{Fe} 4 s$ states are a few volts above $E_{F}$, they contribute appreciably at $5-6 \mathrm{eV}$ below and at $\sim 2.5 \mathrm{eV}$ above $E_{F}$.

Figure 2 shows the valence charge density in the vertical plane cutting through the nearest $\mathrm{Fe}$ and As nuclei. The As sites are easily identified by the presence of two nodes in the radial $4 p$ wave function. Strong covalent bonds between $\mathrm{Fe}$ and As are clearly seen. The filling fraction of the As $p$ states is only $39 \%$, although the extended $p$ orbital spills out of the atomic sphere (2.58 a.u.) somewhat. A fully developed covalent bond corresponds to a filling fraction of $50 \%$. Note that the Pauling electronegativity difference between $\mathrm{Fe}$ and As is only 0.35 so that an almost nonpolar bond is expected.

For a strong covalent bond, the bonding-antibonding splitting is very sensitive to the overlap integrals and, hence, to the distance between the atoms participating in the bond. Figure 1(b) shows the effect of moving As atoms closer to $\mathrm{Fe}$ 

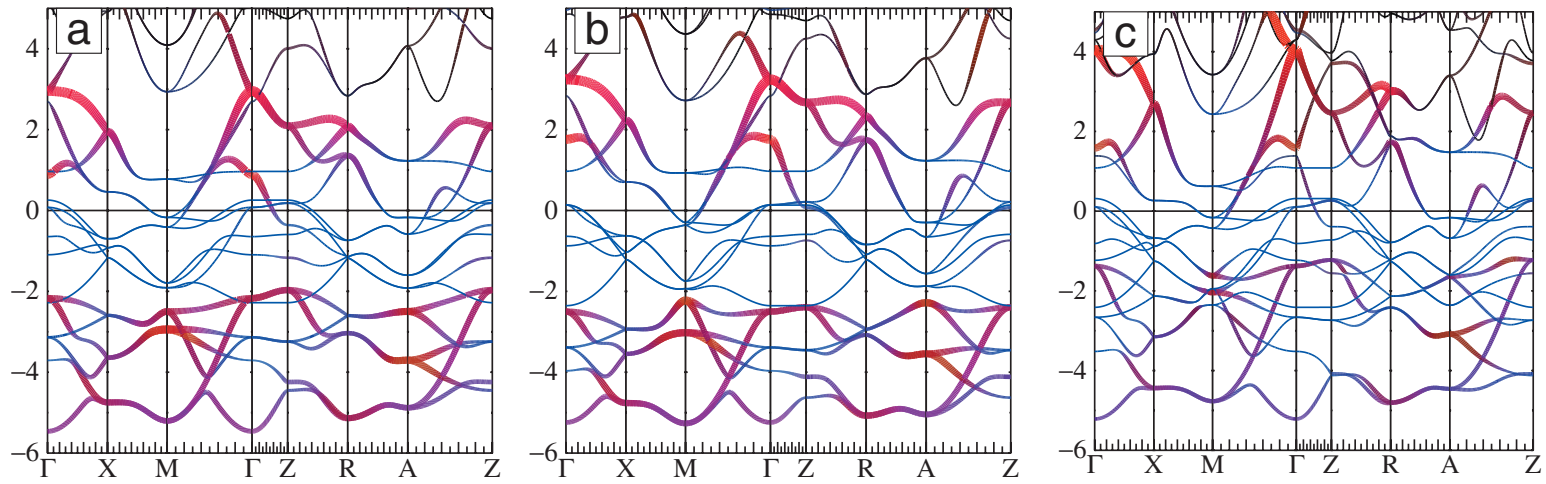

FIG. 1. (Color) Energy bands of LiFeAs in the nonmagnetic state. Mulliken weights of As $(4 p)$ orbitals and Fe $(3 d$ and $4 s)$ orbitals are shown by red and blue color, respectively. Bands with no contributions from these states appear black; fully hybridized bands appear purple. In addition, the As $(4 p)$ weight is shown by line thickness. (a) Experimental lattice parameters with $R_{\mathrm{Fe}-\mathrm{As}}=2.42 \AA$. (b) $R_{\mathrm{Fe}-\mathrm{As}}$ reduced to $2.33 \AA$ A. (c) External potential of $5 \mathrm{eV}$ applied to the As $p$ states (see text).

layers so that $R_{\mathrm{Fe}-\mathrm{As}}$ is reduced from 2.42 to $2.33 \AA$. This relatively small change in $R_{\mathrm{Fe}-\mathrm{As}}$ has a large effect on the band structure. An increase in the bonding-antibonding splitting is evident; the antibonding $\mathrm{Fe}$-As states move notably upward, pulling the Fe partial density of states away from the Fermi level. The main change in the Fermi level is the upward shift of the dispersive band near the $Z$ point, which contains a significant As $p_{z}$ weight. This upward shift of the antibonding states results in the fourfold reduction in the local moment in the stripe ${ }^{16}$ phase (see Table I).

It is useful to view the Fe-As structure as being formed by two As semilayers adsorbed on each side of a freestanding Fe monolayer [which is compressed by 6\% compared to (001) layers in bulk bcc Fe]. The strong hybridization of Fe with As apparent in Figs. 1(a) and 2 appears natural in view of this analogy. In fact, the experimentally measured position of the (chemically similar but slightly smaller) phosphorus adsorbed as a $c(2 \times 2)$ layer on the $\mathrm{Fe}(001)$ surface ${ }^{17}$ translates to the Fe- $P$ bond length of $2.27 \AA$, which is very close to the Fe-As distance in Fe-As compounds. ${ }^{18}$

Surfaces of magnetic transition metals often lose their magnetization under adsorption of such elements as $\mathrm{O}, \mathrm{S}, \mathrm{H}$, N, P, etc. First-principles calculations for such surfaces often show reduced magnetic moments in the surface layer which is strongly bound to the adsorbant; ${ }^{19-21}$ this effect is also observed experimentally. ${ }^{22-24}$ The magnetic "dead layer" on the surface appears precisely due to the chemical bonding, which partially removes the transition-metal $3 d$ states from the Fermi level. Bonding and antibonding surface subbands are common in such cases. ${ }^{25}$ Even if the surface retains some magnetic moment, it may be reduced compared to the bulk. Indeed, as the material is magnetized and the Fermi level reaches the majority-spin antibonding states, further exchange splitting becomes unfavorable. All these arguments fully apply to Fe-As compounds. Although the surface of bcc Fe usually remains magnetic under chemisorption, the local moment in the "freestanding" monolayer in Fe-As compounds is expected to be less stable because it is not supported by the magnetized bulk.

It is reasonable to hypothesize that the emptying of the antibonding states is the primary factor responsible for the equilibrium position of As atoms above the Fe layers. As soon as these states move above the Fermi level, further reduction in $R_{\mathrm{Fe}-\mathrm{As}}$ does not bring a large gain in the binding energy. The proximity of the antibonding Fe-As states to the Fermi level appears to be universal among the iron-pnictide compounds. The analysis presented above indicates that covalent bonding competes with the tendency to form local moments in the $\mathrm{Fe}$ layer. It is therefore quite natural that the Fe magnetic moment is extremely sensitive to $R_{\mathrm{Fe}-\mathrm{As}}$, as noted by other authors. ${ }^{3,26}$ Competition between covalency and magnetism can also explain why band-structure calculations with optimized As positions seem to be in better agreement with experiment compared to those that use experimental As positions. ${ }^{26}$ Indeed, while the local-density approximation or other exchange-correlation potentials used in density-functional theory (DFT) may err in the binding energies, it is reasonable to expect that equilibrium As positions, whether in nature or in DFT, correspond to a similar balance between covalent binding and the tendency to form local moments.

We now concentrate on the magnetic properties of LiFeAs. The so-called stripe phase is believed to be the ground state for other iron-pnictide compounds, ${ }^{16,26}$ we therefore focus on this state. The stabilization energy at the experimental structural parameters is found to be $64 \mathrm{meV}$ per

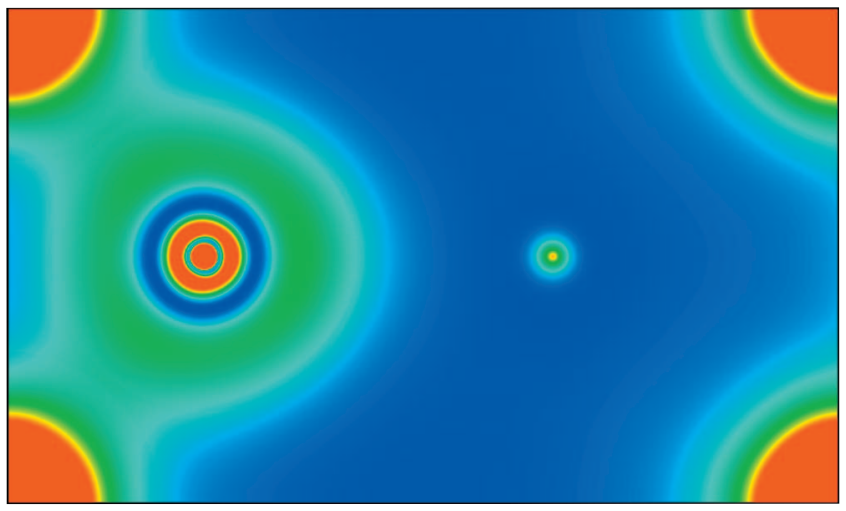

FIG. 2. (Color) Valence charge density (calculated using the FLAPW method) of nonmagnetic LiFeAs in the plane cutting through $\mathrm{Fe}$, As, and Li nuclei. Fe nuclei are at the corners of the plot. The linear color scale extends from 0 (blue) to 0.2 a.u. (red). 
TABLE I. Pair exchange parameters in mRy as a function of $R_{\mathrm{Fe}-\mathrm{As}} . \Delta_{z}$ is the shift of the As layers toward Fe layers given as a fraction of the lattice parameter $c$. Experimental structure corresponds to $\Delta_{z}=0$. $M$ is the local moment in $\mu_{B}$. The exchange parameters $J_{\mathbf{R}}$ are indexed by the crystallographic indices of the connecting vector $\mathbf{R}$ in the simple tetragonal Fe sublattice in which the $x$ axis is aligned parallel to the stripes. A spin pair is parallel if the second index is even and antiparallel otherwise.

\begin{tabular}{lcccccccccccccc}
\hline \hline \multicolumn{1}{c}{$\Delta_{z}$} & $R_{\mathrm{Fe}-\mathrm{As}}$ & $M$ & $J_{100}$ & \multicolumn{1}{c}{$J_{010}$} & \multicolumn{1}{c}{$J_{110}$} & \multicolumn{1}{c}{$J_{200}$} & $J_{020}$ & $J_{120}$ & $J_{210}$ & $J_{001}$ & $J_{101}$ & $J_{011}$ & $J_{0}$ \\
\hline 0 & 2.4204 & 1.28 & -0.83 & -2.61 & -0.56 & -0.24 & -0.23 & 0.27 & -0.036 & -0.063 & -0.024 & -0.035 & 6.7 \\
$0.01 c$ & 2.3972 & 1.05 & -1.26 & -2.96 & -0.75 & -0.19 & -0.14 & 0.30 & -0.033 & -0.053 & -0.039 & -0.049 & 8.1 \\
$0.02 c$ & 2.3739 & 0.81 & -1.73 & -3.11 & -0.91 & 0.03 & 0.09 & 0.35 & -0.009 & -0.044 & -0.044 & -0.057 & 9.8 \\
$0.03 c$ & 2.3517 & 0.57 & -2.10 & -3.10 & -1.01 & 0.22 & 0.34 & 0.38 & 0.025 & -0.032 & -0.061 & -0.052 & 11.6 \\
$0.04 c$ & 2.3294 & 0.31 & -2.43 & -2.90 & -0.94 & 0.63 & 0.60 & 0.36 & 0.18 & -0.032 & -0.016 & -0.028 & 13.6 \\
\hline \hline
\end{tabular}

Fe site in FLAPW or $56 \mathrm{meV}$ in linear muffin-tin orbital (LMTO) method; the local moment is $1.40 \mu_{B}$ in FLAPW or $1.49 \mu_{B}$ in LMTO. Further analysis shows that the trends in the exchange interaction in the stripe phase are insensitive to the particular choice of ASA parameters; the following results are, therefore, quite robust.

Among the possible exchange mechanisms, superexchange and Fermi surface nesting between electron and hole sheets are usually mentioned for Fe-As compounds. ${ }^{16,26} \mathrm{We}$ calculated the pair exchange parameters for LiFeAs using the linear-response technique ${ }^{27}$ with a subsequent division by $\mathbf{S}_{i} \mathbf{S}_{j}$, where $\mathbf{S}_{i}$ is the total spin moment in the atomic sphere at site $i$. These parameters map the energies of small deviations from the reference state to the Heisenberg model $E$ $=E_{0}-\sum_{i j} J_{i j} S_{i} S_{j}$. They were analyzed as a function of $R_{\mathrm{Fe}-\mathrm{As}}$, doping level, and also of the fictitious external potential $V$ coupled to the occupation $n_{p}$ of the As $p$ orbital (see below).

The calculated pair exchange parameters for several nearest neighbors (NNs) in the stripe phase are listed in Tables I and II. The tables also include the values $J_{0}=\Sigma_{i} p_{0 i} J_{0 i}$ where $p_{i j}=1$ or -1 for parallel and antiparallel spin pairs, respectively; and $J_{0}$ is proportional to the Weiss field.

The exchange interaction is quite long-range; some parameters for pairs beyond those included in the table are comparable with, say, $J_{020}$. The contribution of the first two coordination spheres to $J_{0}$ declines steadily from 0.86 at $\Delta_{z}$ $=0$ to 0.35 at $\Delta_{z}=0.04 c$ (Table I). A similar but weaker trend is observed when $V$ is increased (Table II). Thus, the reduction in the local moment is accompanied by the increase in the interaction range. Further, the ratio $J_{0} / J_{00}$, where $J_{00}$ is defined similar to $J_{i j}$ and reflects the magnitude of the on-site (Hund) exchange, steadily grows from 0.26 at $\Delta_{z}=0$ to 0.70 at $\Delta_{z}=0.04 c$. These trends indicate that the degree of itinerancy significantly increases as $R_{\mathrm{Fe}-\mathrm{As}}$ is decreased.

When the local moment is small, the exchange parameters are isotropic, as required by symmetry. For larger moments there is a large anisotropy, in particular, for NNs. For all Fe-As distances (Table I) the NN and next-nearest-neighbor (NNN) exchange parameters are antiferromagnetic (AFM); the exchange along the stripe is thus frustrated. The ratio $J_{110} / J_{100}$ decreases from 0.68 at $\Delta_{z}=0$ (see Table I) to 0.39 at $\Delta_{z}=0.04 c$; this trend tends to make the stripe phase less stable.

We now discuss the dependence of exchange coupling on the external potential $V$ coupled to the occupation of the As $p$ states. This is done by adding an additional term $V n_{p}$ to the Hamiltonian; DFT self-consistency is achieved for each value of $V$. Adding $V$ results in the raising of both bonding and antibonding Fe-As states so that the antibonding states are moved away from the Fermi level. ${ }^{28}$ This change is expected to have a strong effect on the magnitude of the superexchange interaction. ${ }^{29}$ Figure 1(c) shows the energy bands obtained with $V=5 \mathrm{eV}$. The band structure close to the Fermi level is very similar to $V=0$ (Fig. 1). Therefore, direct

TABLE II. Pair exchange parameters as a function of the external potential $V$ coupled to As $p$ occupation (see text). The last four lines are for $V=0$ with the doping level per f.u. listed in the first column. The notation is the same as in Table I.

\begin{tabular}{cccccccccccccc}
\hline \hline$V$ & $M$ & $J_{100}$ & \multicolumn{1}{c}{$J_{010}$} & $J_{110}$ & $J_{200}$ & $J_{020}$ & $J_{120}$ & $J_{210}$ & $J_{001}$ & $J_{101}$ & $J_{011}$ & $J_{0}$ \\
\hline$-1 \mathrm{eV}$ & 1.52 & -0.64 & -2.68 & -0.46 & -0.20 & -0.13 & 0.33 & 0.017 & -0.087 & -0.030 & -0.044 & 6.3 \\
0 & 1.28 & -0.83 & -2.61 & -0.56 & -0.24 & -0.23 & 0.27 & -0.036 & -0.063 & -0.024 & -0.035 & 6.7 \\
$1 \mathrm{eV}$ & 1.10 & -0.70 & -2.30 & -0.65 & -0.14 & -0.22 & 0.24 & -0.068 & -0.060 & -0.037 & -0.052 & 7.5 \\
$2 \mathrm{eV}$ & 0.96 & -0.43 & -1.86 & -0.71 & -0.019 & -0.22 & 0.21 & -0.089 & -0.058 & -0.048 & -0.073 & 8.0 \\
$3 \mathrm{eV}$ & 0.84 & -0.06 & -1.33 & -0.78 & 0.051 & -0.25 & 0.16 & -0.13 & -0.040 & -0.060 & -0.097 & 8.1 \\
$4 \mathrm{eV}$ & 0.70 & 0.30 & -0.65 & -0.87 & -0.059 & -0.32 & 0.033 & -0.25 & -0.0082 & -0.068 & -0.122 & 7.7 \\
$5 \mathrm{eV}$ & 0.56 & 0.84 & 0.30 & -0.79 & -0.19 & -0.27 & -0.087 & -0.33 & -0.0013 & -0.121 & -0.154 & 6.6 \\
$-0.2 e$ & 1.20 & -1.24 & -1.26 & -0.63 & -0.12 & -0.49 & 0.12 & -0.30 & 0.052 & -0.028 & -0.005 & 3.0 \\
$-0.1 e$ & 1.22 & -1.19 & -2.13 & -0.54 & -0.09 & -0.29 & 0.34 & -0.19 & -0.042 & -0.034 & -0.052 & 5.3 \\
$+0.1 e$ & 1.34 & -0.56 & -2.80 & -0.71 & -0.35 & -0.38 & 0.15 & -0.02 & -0.011 & -0.0042 & -0.013 & 7.0 \\
$+0.2 e$ & 1.40 & -0.41 & -2.65 & -0.88 & -0.41 & -0.49 & -0.038 & -0.01 & 0.003 & -0.022 & -0.027 & 6.7 \\
\hline \hline
\end{tabular}


exchange (including the "nesting" contribution) should be insensitive to $V$. Thus, the analysis of trends associated with the As $p$ level shift will allow us to disentangle direct metallic exchange from superexchange contributions.

Table II shows that the increase in $V$ leads to the decrease and eventual sign change in the NN coupling to ferromagnetic. The NNN exchange, however, is almost unaffected. This result suggests that NN exchange has a large AFM contribution from superexchange, while $J_{110}$ is primarily due to direct metallic exchange including the nesting effects. Moreover, the direct exchange contribution to the NN exchange appears to be ferromagnetic.

Table II also shows the dependence of the exchange parameters on the doping level, which was changed by varying the charge in the Li nuclei. The local moment is quite insensitive to doping of $\pm 20 \%$, but a drastic change in exchange parameters is apparent. Most notably, the anisotropy of NN exchange interaction is reduced from $J_{100} / J_{010} \approx 1$ at $20 \%$ hole doping to 0.15 at $20 \%$ electron doping. Also, the ratio $J_{110} / J_{100}$, which is important for the stability of the stripe phase, increases from about 0.5 to 2.1 . In the stripe phase the exchange splitting brings the Fermi level close to the antibonding states. It is likely that the anisotropy of NN coupling reflects the sensitivity of superexchange to the anisotropy of the electronic structure. Interestingly, $J_{100}$ is much more sensitive to both the Fe-As distance and doping than $J_{010}$.

The exchange parameters between the Fe layers are quite small and frustrated for the stripe phase (the first three shown in the Tables I and II are all AFM). These parameters likely vary between different Fe-As compounds.

In conclusion, layered iron-pnictide compounds are characterized by strong covalent Fe-As bonding which is antagonistic to magnetism and responsible for the huge magnetostructural coupling. The magnetic interaction is long range and shows increasing itinerancy with decreasing $R_{\mathrm{Fe}-\mathrm{As}}$. Superexchange makes a dominant AFM contribution to the NN magnetic coupling, while the NNN coupling is mainly due to direct exchange. The anisotropy of NN coupling is sensitive to doping.

We thank Mark van Schilfgaarde for the use of his LMTO codes and Igor Mazin for useful comments. K.D.B. is a Cottrell Scholar of Research Corporation. Support from the Nebraska Research Initiative is acknowledged. Work at Ames Laboratory was supported by Basic Energy Sciences, Department of Energy under Contract No. DE-AC02-07CH11358.
${ }^{1}$ Y. Kamihara, T. Watanabe, M. Hirano, and H. Hosono, J. Am. Chem. Soc. 130, 3296 (2008).

${ }^{2}$ I. I. Mazin, D. J. Singh, M. D. Johannes, and M. H. Du, Phys. Rev. Lett. 101, 057003 (2008).

${ }^{3}$ Z. P. Yin, S. Lebègue, M. J. Han, B. P. Neal, S. Y. Savrasov, and W. E. Pickett, Phys. Rev. Lett. 101, 047001 (2008).

${ }^{4}$ C. Cao, P. J. Hirschfeld, and H.-P. Cheng, Phys. Rev. B 77, 220506(R) (2008).

${ }^{5}$ V. Cvetkovic and Z. Tesanovic, arXiv:0804.4678 (unpublished).

${ }^{6} \mathrm{~J}$. Wu, P. Phillips, and A. H. Castro Neto, Phys. Rev. Lett. 101, 126401 (2008).

${ }^{7}$ S. Mirbt, B. Sanyal, C. Isheden, and B. Johansson, Phys. Rev. B 67, 155421 (2003).

${ }^{8}$ L. Boeri, O. V. Dolgov, and A. A. Golubov, Phys. Rev. Lett. 101, 026403 (2008).

${ }^{9}$ T. Yildirim, arXiv:0807:3936 (unpublished).

${ }^{10}$ D. J. Singh and M. H. Du, Phys. Rev. Lett. 100, 237003 (2008).

${ }^{11}$ V. Vildosola, L. Pourovskii, R. Arita, S. Biermann, and A. Georges, Phys. Rev. B 78, 064518 (2008).

${ }^{12}$ X. C. Wang, Q. Q. Liu, Y. X. Lv, W. B. Gao, L. X. Yang, R. C. Yu, F. Y. Li, and C. Q. Jin, Solid State Commun. 148, 538 (2008).

${ }^{13}$ D. J. Singh, Phys. Rev. B 78, 094511 (2008).

${ }^{14} \mathrm{We}$ used the atomic sphere approximation of the tight-binding linear muffin-tin orbital method. The empty spheres, the sphere radii, and the basis set were very carefully chosen to provide energy bands in close agreement with the full-potential linear augmented plane-wave method. (We used the FLEUR implementation; see www.flapw.de) We also verified that the energy bands evolve similar to FLAPW bands when $R_{\mathrm{Fe}-\mathrm{As}}$ is reduced. Some results, where noted, were obtained using FLAPW.
${ }^{15}$ J. H. Tapp, Z. Tang, B. Lv, K. Sasmal, B. Lorenz, P. C. W. Chu, and A. M. Guloy, Phys. Rev. B. 78, 060505(R) (2008).

${ }^{16}$ T. Yildirim, Phys. Rev. Lett. 101, 057010 (2008).

${ }^{17}$ W. R. A. Huff, Y. Chen, X. S. Zhang, L. J. Terminello, F. M. Tao, and Y. K. Pan, Phys. Rev. B 55, 10830 (1997).

${ }^{18}$ Interestingly, another superconductor $\mathrm{Na}_{x} \mathrm{CoO}_{2}$ also has widely separated hexagonal $\mathrm{Co}$ monolayers with a monolayer of $\mathrm{O}$ internally adsorbed on each side; the Co-O distance there is very close to that for the Co surface with an adsorbed $\mathrm{O}$ monolayer.

${ }^{19}$ M. Weinert and J. W. Davenport, Phys. Rev. Lett. 54, 1547 (1985).

${ }^{20}$ S. R. Chubb and W. E. Pickett, Phys. Rev. B 38, 10227 (1988).

${ }^{21}$ W. T. Geng, A. J. Freeman, and R. Q. Wu, Phys. Rev. B 63, 064427 (2001).

${ }^{22}$ M. Landolt and M. Campagna, Phys. Rev. Lett. 39, 568 (1977).

${ }^{23}$ F. Passek and M. Donath, Phys. Rev. Lett. 71, 2122 (1993).

${ }^{24}$ P. Morrall, F. Schedin, L. Hewitt, C. A. Muryn, V. R. Dhanak, and G. Thornton, Phys. Rev. B 64, 064407 (2001).

${ }^{25}$ K. D. Belashchenko, E. Y. Tsymbal, M. van Schilfgaarde, D. A. Stewart, I. I. Oleinik, and S. S. Jaswal, Phys. Rev. B 69, 174408 (2004).

${ }^{26}$ I. I. Mazin, M. D. Johannes, L. Boeri, K. Koepernik, and D. J. Singh, Phys. Rev. B 78, 085104 (2008).

${ }^{27}$ M. van Schilfgaarde and V. P. Antropov, J. Appl. Phys. 85, 4827 (1999); V. P. Antropov, M. van Schilfgaarde, S. Brink, and J. L. $\mathrm{Xu}$, ibid. 99, 08F507 (2006).

${ }^{28}$ The self-consistent shift of the As $p$ states is much smaller than $V$ due to electrostatic screening.

${ }^{29}$ The term superexchange is used loosely here to denote any kind of magnetic coupling involving Fe-As hopping; direct exchange refers to all other mechanisms. 